\title{
Mini review: Multielectrode recordings in insect brains
}

\author{
Mit Balvantray Bhavsar, Ralf Heinrich, Andreas Stumpner \\ Georg-August-University of Göttingen, Johann-Friedrich-Blumenbach-Institute for Zoology and Anthropology, Department of \\ Cellular Neurobiology, Julia-Lermontowa-Weg 3, D-37077 Göttingen, Germany
}

Correspondence: Mit Balvantray Bhavsar

E-mail: mbhavsa@gwdg.de

Received: October 29, 2015

Published online: December 21, 2015

\begin{abstract}
Currently, more and more laboratories are acquiring the capability of simultaneously detecting the extracellular activity of neurons in anaesthetized and awake animals by multielectrode recordings. In insects, multielectrode recordings are challenging due to the small size of the nervous system. Nevertheless, multielectrode recordings have been successfully established in brains of cockroaches, honeybees, fruit flies and grasshoppers to study sensory processing related to mechanosensation, olfaction, vision and audition. The number of neurons which can be recorded using such multielectrodes did not exceed 5 and likely depends on factors like recorded compartment of the neuron, impedance of the multielectrode, number of wires included in the multielectrode and threshold for spike detection. Signal-to-noise ratio (SNR) of the recordings obviously depends on the material and method used for the production of multielectrodes. To mark the location of the recording, different methods like current-driven copper deposition, labelling with fluorescent dye and electrocoagulation of nervous tissue are used. As expected, multielectrode recordings are more difficult in freely moving compared to restrained insects due to movement artifacts and requirement for fixed placement of the multielectrode at a particular recording site in the central nervous system (CNS). Specific characteristics of different preparations and sensory systems that include, disentangling spike collisions connected to auditory stimulation, increase in SNR after extended recording periods in olfactory systems and photoelectrical effects from compound eyes associated with visual stimulation, may require special attention and particular adaptations.
\end{abstract}

To cite this article: Mit Balvantray Bhavsar, et al. Mini review: multielectrode recordings in insect brains. Neurosci Commun 2015; 1: e1088. doi: 10.14800/nc.1088.

Copyright: () 2015 The Authors. Licensed under a Creative Commons Attribution 4.0 International License which allows users including authors of articles to copy and redistribute the material in any medium or format, in addition to remix, transform, and build upon the material for any purpose, even commercially, as long as the author and original source are properly cited or credited.

\section{Introduction}

One of the major developments in the field of neurophysiology is the use of multielectrodes (or tetrodes in case of four wires) to simultaneously monitor spiking activity of populations of neurons $[1,2]$ which is used to study fundamental aspects of the functional organization of the nervous system. Long term multielectrode recordings have become routine in mammalian neurophysiology ${ }^{[3,4]}$ and at present a large variety of experimental conditions are applied, which include in-vitro preparations using culture or brain slices ${ }^{[5,6]}$, acute and chronic recordings in anesthetized animals ${ }^{[7]}$, long term recordings in behaving animals ${ }^{[8]}$ and even short term neurophysiological monitoring in human subjects ${ }^{[9]}$. However, in insects multielectrode recordings still remain a challenge due to the smaller size of their nervous systems. Intracellular recordings with sharp electrodes are very popular in insects since they provide physiological and morphological data on identified neurons. However, this technique is usually limited to one cell at a 
time (very rarely more using additional glass electrodes), requires a restrained animal and can typically only be stabilized for relatively short periods of time.

Over the last decade considerable attention has been directed on how populations of neurons encode and process different aspects of a sensory stimulus in insects. So far, intracellular recording of single units in several individuals or in one individual successively is a widely used method to extrapolate their potential combined activity ${ }^{[10,11]}$. However, this method suffers from several limitations like trial-to-trial and interindividual neuronal variability ${ }^{[12]}$. In order to analyze the activity of populations of neurons, however, it would be more appropriate to record the activity of several neurons at the same time in the same individual [13]. Multiunit recordings using combined single wires (multielectrodes) can serve this purpose. They allow recording the activity of multiple neurons from a particular recording site via a bundle of 3-4 closely associated insulated microwires. Association of recorded spikes with particular neurons relies on the fact that both amplitude and shape of spikes change with distance and tissue conductivity between the electrode tip and the cell ${ }^{[14,15]}$. Since every cell will be at a different distance from the different contact sites of the electrodes, its activity will be recorded with a different shape and amplitude in each channel of the multielectrode. Recordings with this method are typically much more stable than intracellular recordings ${ }^{[13]}$ allowing for long term analysis of the recorded units. In insects, few research groups have successfully used this method for studying mechanosensory processing in cockroaches [16], olfactory processing in honeybees [17-20] and locusts [21, 22], visual processing in fruit flies [23] and auditory processing in grasshoppers ${ }^{[13]}$. Here we discuss the methods applied when studying different sensory systems and the adaptations they had to make to solve specific problems with using multielectrode recordings in small insects.

\section{Type of material used for production of multielectrodes}

Multielectrodes are typically made from copper, tungsten or nickel-chromium (NiCr) wires. However, signal-to-noise ratio of the recordings differs when using these different metals for multielectrodes. Signal-to-noise ratio (SNR) generally means the dimensionless ratio of signal power to noise power. It allows quantifying the size of signal relative to the fluctuations (called noise) that are unrelated to neural spiking activity and outside experimental control [24]. High SNR determines the quality of electrophysiological recordings and the ability to extract and sort single spikes from background noise. The SNR depends on factors like impedance and diameter of the multielectrode, recorded compartment of the neurons and the distance between the neuron and the tip of the electrode. Among these, the impedance of the multielectrode is likely the most influencing factor. It has been shown that for example $12 \mu \mathrm{m}$ tungsten wires gave better SNR ( $>2)$ than $15 \mu \mathrm{m}$ copper wires $(<1.5)$ which is likely due to the lower resistance of tungsten wires $(40-70 \mathrm{k} \Omega$ ) compared to copper wires $(200-700 \mathrm{k} \Omega)^{[13]}$. Additionally, higher tensile strength of the tungsten wires compared to copper wires eases penetration of the tissue and enables the repeated use of the same multielectrode in multiple experiments.

Another method to increase SNR in multielectrode recordings is electroplating the recording tip of the multielectrode using noble metals like iridium, gold or platinum. Such an electroplating process basically uses current to reduce dissolved metal cations so that they form a noble metal coating on the electrode. One study ${ }^{[23]}$ has used iridium oxide $\left(\mathrm{IrO}_{2}\right)$ films which were deposited at the tip of four $17 \mu \mathrm{m}$ insulated $\mathrm{NiCr}$ wires by an anodic coating process with an electrochemical work station at room temperature. The impedance of the $\mathrm{IrO}_{2}$ modified electrode was reduced to almost $10 \%$ compared to the unmodified $\mathrm{NiCr}$ multielectrode. Benefitting from the decreased impedance of the electrodeposited $\mathrm{IrO}_{2}$ films, the SNR was increased to 7.5 which was the highest SNR described in all studies considered here. However, one of the pitfalls of this method is that it is required to plate the multielectrode again after each use because of the low durability of the plating metal ${ }^{[25]}$. Another method to decrease the impedance of multielectrodes is by changing their charge capacity for example with the equipment NanoZ (Neuralynx - Bozeman, USA). It increases the charge capacity of the multielectrode by passing bipolar, constant current square waves to each wire of the multielectrode ${ }^{[26]}$. The average decrease in resistance of multielectrodes after using NanoZ was 30\% from the original resistance ${ }^{[13]}$.

One point of emphasis during production of multielectrodes is the fragile nature of these microwires. They are easily bent or damaged if not carefully handled during production and implantation [13, 26]. Wires may be carefully retracted from the preparation after the experiment is completed, allowing for two or three uses of the same multielectrode. After each use the tip must be cut or cleaned in an ultrasound waterbath to remove any deposits and plating needs to be refreshed before each use ${ }^{[27]}$.

\section{Number of neurons that can be recorded using multielectrodes}

Up to five neurons have been simultaneously recorded with sufficient quality to be isolated as one unit [18, 23] (Bhavsar unpublished). This number depends on different 
factors like the recorded compartment of the neuron, impedance of the multielectrode, number of wires of the multielectrode and threshold of spike sorting. For example, in the grasshopper auditory system around 20 auditory neurons ascending from the thorax form a bundle that enters each hemisphere of the brain ${ }^{[28,29]}$. Since one can record large amplitude action potential from the large axons of ascending auditory neurons, it possible to detect up to five units using multielectrodes with good SNR. Recording electrical activity from smaller dendrites in a neuropile may include signals from a high number of cells, but SNR will be low. Another important factor affecting the number of simultaneously recorded neurons is the impedance of the multielectrode. In the visual system of the fruit fly Drosophila melanogaster it was possible to record from 5 different neurons using low impedance fabricated $\mathrm{IrO}_{2}$ stereotrodes suggesting improved performance of multielectrodes after $\mathrm{IrO}_{2}$ deposition [23]. The number of neurons that can be distinguished also depends on the number of wires included in the multielectrode. It is obvious that with a larger number of wires, more neurons can be detected. For insect brains it is advisable to use only 3 to 4 wires per multielectrode, since with increasing number of wires the total diameter of the tip also increases which causes larger damage to the nervous tissue during electrode insertion. This is seen especially in very small brains like those of Drosophila melanogaster (left-right diameter 600 $\mu \mathrm{m})$ where stereotrodes made of $\mathrm{NiCr}$ wires having a total diameter of at least $34 \mu \mathrm{m}$ were described as being too large for recording due to insertion damage ( $\mathrm{Lu} \mathrm{Yi} \mathrm{-} \mathrm{personal}$ communication), even though recordings contained good SNR ${ }^{[23]}$. Threshold of spike detection during spike sorting is a further important factor for detecting spikes of different units from the recording. A common criterion has been established to choose the threshold as a multiple of an estimate of the standard deviation (SD) of the noise (noise includes any activity that is not related to the stimulus), i.e., threshold $=$ mean $( \pm) \mathrm{k} * \mathrm{SD}$, where $\mathrm{k}$ is a constant ${ }^{[30]}$. Choosing the value of $\mathrm{k}$ is critical: if the value is too low, noise fluctuations will be interpreted as spikes and if it is too high, too many low amplitude spikes will be missed which eventually decreases the number of detected units. A value of $\mathrm{k}=3$ provided the best compromise between avoidance of noise fluctuations and detection of spikes in honeybees and grasshoppers ${ }^{[13,18]}$. However, higher values like $\mathrm{k}=4.5$ have also provided satisfactory results in fruit flies due to the high SNR of the recordings ${ }^{[23]}$.

\section{Methods to mark the location of the recordings}

One of the major limitations of multielectrode recordings is that the morphology of the recorded neurons remains unknown unlike in intracellularly recorded neurons which can be filled with dye following physiological characterization. However, there are some methods available to at least mark the position of the tip of the multielectrode. Such information about the location of the recording can be used to search for neurons with sharp electrodes and fill them with dye during intracellular recordings in follow up experiments. For the visualization of the recording site after a successful extracellular recording, three methods have been used, each with particular merits and demerits.

The first method is electrical current-driven deposition of copper from copper plated $\mathrm{NiCr}$ wires ${ }^{[31,32]}$. At the end of the recording experiment, a $10 \mu \mathrm{A}$ and $5 \mathrm{~ms}$ DC current pulses was passed between one of the tetrode wires and the reference electrode to deposit copper at the recording site. Concentrated brownish deposits occurring in several adjacent serial sections were identified as the recording location. This method was also attempted with unplated copper wires on the grasshopper brain [13]. However, results were not reproducible. A likely explanation for the failure to detect copper deposits in the brain tissue is that the maximum current which can be passed via such small diameter unplated copper wires did not dissociate sufficient copper at the recording site. Another explanation could be that copper dissolves less effectively from solid wire than from a thin layer of copper plating at the tip of the multielectrode.

A second method for marking the recording location is the application of fluorescent dyes like Alexa hydrazide [17], green fluorescent Nissl stain ${ }^{[23]}$ or lucifer yellow [13]. In grasshoppers, after a successful recording, the multielectrode was retracted from the brain, dipped into the fluorescent dye and reinserted to the previous location until the auditory activity was detected again ${ }^{[13]}$. The electrode was kept at this position for $10 \mathrm{~s}$ to let the dye diffuse into the tissue. Then with the electrode still in place, a drop of paraformaldehyde (PFA, 4\%) was added to fix the dye in the tissue and prevent extensive diffusion of the dye. This method includes some uncertainty about the exact recording site since the electrode was removed for dipping in the fluorescent dye and then reinserted. However, in the grasshopper brain recording of auditory activity was re-established immediately in all preparations suggesting that the multielectrode was reintroduced to its previous recording position with reasonable precision ${ }^{[13]}$.

The third method is electric coagulation of brain tissue by passing high current for several minutes, either between one of the tungsten wires and the reference wire ${ }^{[13]}$ or between two tungsten wires (Bhavsar unpublished). The high current $(100 \mu \mathrm{A})$ coagulates the brain tissue and generates a black spot at the tissue surrounding the tip of the electrode. This method harbors the risk of damaging a larger volume of 
tissue around the electrode which may go along with shrinkage and deformation. So one needs to control the time of current injection to have reliable results without destroying larger parts of the brain. The appearance of gas bubbles at the deposition site may serve as a good indicator for sufficient current injection. A clear advantage of this method (and also copper deposition - see above), compared to the introduction of dye with the multielectrodes is that the electrode position is not changed between recording and staining.

\section{Recording in freely moving insects vs recording in restrained insects}

Obviously, multielectrode recordings in freely moving insects are more challenging than in restrained insects, since small movements of the brain relative to the electrode impair the recording. Nevertheless, recordings from unstrained insects have been successfully performed in honeybees ${ }^{[19,20]}$ and cockroaches ${ }^{[26,33]}$. Most importantly after insertion, the multielectrode must be attached to the nervous system in order to stay at the same position during the subsequent recording period. For this, two component silicon glue has been applied into the head capsule ${ }^{[19,20]}$. It turned out to be important to remove the hemolymph surrounding the brain before applying the glue to enable quick solidification ${ }^{[19]}$. Another option for fixing the multielectrode at the recording site is to use hot dental wax ${ }^{[27]}$. The number of wires used to produce multielectrodes also affects the recording in freely moving insects. For example, in one study honey bees were not moving naturally with tetrodes, since these were too heavy ${ }^{[19]}$. Therefore, the number of wires in a multielectrode was reduced to two in that study. Also the length of the multielectrode must be adjusted in a way that the animal can move freely in the setup without restrictions and without getting entangled in the wires [27]. In recordings from mechanosensory units from the central complex of 50 freely moving cockroaches [27] none of the experiments was terminated because the cockroach damaged the wire sets. Nowadays, wireless electrophysiological systems are commercially available which could be more appropriate for small freely moving animals ${ }^{[34,35]}$, but they suffer from limitations like speed of data acquisition, battery life and weight ${ }^{[27]}$.

In comparison to freely moving insects, the multielectrode recordings in restrained insects are relatively easy and straightforward. The animal can be anesthetized for fixation and appendages may be cut or fixed to a holder to reduce movements ${ }^{[17]}$. Before the insertion of the multielectrode, the brain is typically supported by a metal platform ${ }^{[13,21]}$ to reduce its movements as much as possible. Restrained animals offer the possibility to record from more than one area of the brain in the same animal (either simultaneously or successively) provided that the electrode insertion induces only minor damage. As useful as these restrained preparations have been and will continue to be, they do present some limitations. Numerous studies have demonstrated that sensory processing depends on the behavioral state and can be altered by restraint. In such restrained conditions the behaviors that the insect can perform - if at all - are limited as well. Most restrained preparations are "open loop", that is, they do not allow for normal movement-related feedback to the system ${ }^{[27]}$.

\section{Specific differences between species and sensory systems}

Multielectrode recordings in insects were mainly used in studies of sensory processing (see introduction). Specific differences that affect the analysis of data from multielectrode recordings have been detected in the processing of information from different sensory modalities. For example, while recording from olfactory neurons in a honeybee ${ }^{[18]}$, the SNR of the recording improved over 15-30 minutes after inserting the multielectrode in the brain. This is due to the fact that the hemolymph or saline surrounding the brain can prevent the electrodes from making a tight contact with glia and neurons. As time passes, the saline evaporates and the electrodes get closer to the neurons which will eventually increase SNR. Such an effect has not been observed in the grasshopper brain ${ }^{[13]}$.

Auditory neurons tend to fire synchronously especially at the beginning of an acoustic stimulus because of the tight coupling between the response and the temporally precise stimuli [13]. Synchronous spiking leads to recording of complex spike shapes (= collisions) due to spike overlap. Therefore, it is equally important to extract such spike collision from the recording to reduce the loss of important information ${ }^{[36]}$. Surprisingly, spike collision analysis was not performed in most studies on non-auditory sensory systems. The likely reason is, that spike collisions are not as frequent in other systems as in auditory systems due to less precise coupling of activity of several neurons to a stimulus (Roy Ritzmann - personal communication). Nevertheless, the importance of extracting overlapping spikes should not be overlooked since it may provide additional useful information.

A study on olfactory processing in honey bees used differential (pairwise subtracted) channels for the spike sorting that resulted in reduced noise without compromising spike detection too much ${ }^{[18]}$. However, this is helpful only when the stimulus related activities recorded by individual channels of the multielectrode are sufficiently different. In cases of similar activity recorded by different channels (see 


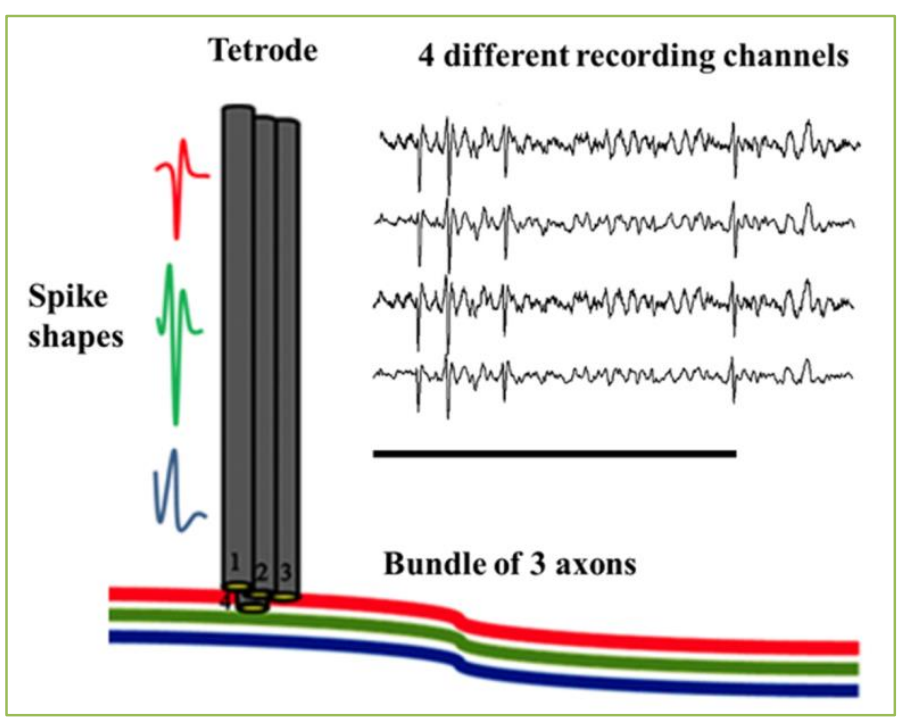

Figure 1. Schematic drawing of a multielectrode (Tetrode with 4 wires) placed near the bundle of 3 axons in invertebrate nervous tissue. The differences between the channels are typically subtle. The black line represents the stimulus. The closer the recorded neurons are positioned to the electrode, the stronger and better signal to noise ratios can be achieved. What ultimately leads to principle differences in spike shape is not known.

fig.1), subtraction could also reduce the specific stimulus-related activity and hence is not recommended.

A study on visual processing in fruit flies observed a special problem related to optical stimulation, a photoelectrical effect while recording with multielectrodes from the compound eye of the fruit fly Drosophila melanogaster ${ }^{[23]}$. During stimulation with blue light, the metals (NiCr) used for the production of the multielectrode emit electrons when light shines upon them which affect the potential of the photoreceptors.

\section{Conclusions}

Here, the pros and cons of multielectrode recordings in different sensory systems of insects were reviewed. Lower resistance wires like tungsten or gold plated $\mathrm{NiCr}$ are probably best suited for multielectrode recordings. Compared to other physiological recording techniques in insects, multielectrode recordings ensure long-time access to population neuronal activity in behaving insects. Multielectrode recordings can also be successfully applied to freely moving insects. A major pitfall is missing neuronal identification as compared to intracellular studies. In the ideal case, both methods could be combined in the same species and may allow identification of neurons based on their physiology also in multielectrode recordings. Interindividual neuronal variability, however, may be the main obstacle in this approach.

\section{Conflicting interests}

The authors have declared that no competing interests exist.

\section{Acknowledgements}

We acknowledge Prof. Dr. Roy Ritzmann (Case Western Reserve University, Cleveland, Ohio, United States), Prof. Dr. Lu Yi (Chinese Academy of Sciences, Beijing, China) and Dr. Martin Brill (Cold Spring Harbor Laboratory, Cold Spring Harbor, United States) for personal communications sharing the experiences on multielectrode recordings in cockroaches, fruit flies and honey bees.

\section{References}

1. Wise KD, Angell JB. A low capacitance multielectrode probe for use in extracellular neurophysiology. IEEE Trans Biomed Eng 1975; 22: 212-220.

2. Recce and O'Keefe. The tetrode: An improved technique for multi-unit extracellular recording. Soc Neurosci 1989; 15: 490.

3. Nicolelis MA, Lin RC, Woodward DJ, Chapin JK. Induction of immediate spatiotemporal changes in thalamic networks by peripheral block of ascending cutaneous information. Nature 1993; 361: 533-536.

4. Welsh JP, Lang EJ, Suglhara I, Llinás R. Dynamic organization of motor control within the olivocerebellar system. Nature 1995; 374 : 453-457.

5. Gross GW, Williams a N, Lucas JH. Recording of spontaneous activity with photoetched microelectrode surfaces from mouse spinal neurons in culture. J Neurosci Methods 1982; 5: 13-22.

6. Potter SM. Distributed processing in cultured neuronal networks. Prog Brain Res 2001; 130: 49-62.

7. Ghazanfar AA, Nicolelis MA. Nonlinear processing of tactile information in the thalamocortical loop. J Neurophysiol 1997; 78: 506-510.

8. Laubach M, Wessberg J, Nicolelis MA. Cortical ensemble activity increasingly predicts behaviour outcomes during learning of a motor task. Nature 2000; 405: 567-571.

9. Kreiman G, Koch C, Fried I. Imagery neurons in the human brain. Nature 2000; 408: 357-361.

10. Schöneich S, Kostarakos K, Hedwig B. An auditory feature detection circuit for sound pattern recognition. Sci Adv 2015; 1: e1500325-e1500325.

11. Meckenhäuser G, Krämer S, Farkhooi F, Ronacher B, Nawrot MP. Neural representation of calling songs and their behavioral relevance in the grasshopper auditory system. Front Syst Neurosci 2014; 883: 1-12.

12. Ronacher B, Franz A, Wohlgemuth S, Hennig RM. Variability of spike trains and the processing of temporal patterns of acoustic signals - Problems, constraints, and solutions. J Comp Physiol A Neuroethol Sensory, Neural, Behav Physiol 2004; 190: 257-277.

13. Bhavsar MB, Heinrich R, Stumpner A. Multielectrode recordings from auditory neurons in the brain of a small grasshopper. $\mathrm{J}$ 
Neurosci Methods 2015; 256: 63-73.

14. Gray CM, Maldonado PE, Wilson M, McNaughton B. Tetrodes markedly improve the reliability and yield of multiple single-unit isolation from multi-unit recordings in cat striate cortex. J Neurosci Methods 1995; 63: 43-54.

15. Harris KD, Henze DA, Csicsvari J, Hirase H, Buzsáki G. Accuracy of tetrode spike separation as determined by simultaneous intracellular and extracellular measurements. J Neurophysiol 2000; 84: 401-414.

16. Ritzmann RE, Ridgel AL, Pollack AJ. Multi-unit recording of antennal mechano-sensitive units in the central complex of the cockroach, Blaberus discoidalis. J Comp Physiol A - Neuroethol Sensory, Neural, Behav Physiol 2008; 194: 341-360.

17. Brill MF, Reuter M, Rössler W, Strube-Bloss MF. Simultaneous long-term recordings at two neuronal processing stages in behaving honeybees. J Vis Exp 2014; 89: e51750

18. Brill MF, Rosenbaum T, Reus I, Kleineidam CJ, Nawrot MP, Rössler W. Parallel processing via a dual olfactory pathway in the honeybee. J Neurosci 2013; 33: 2443-2456.

19. Duer A, Paffhausen BH, Menzel R. High order neural correlates of social behavior in the honeybee brain. J Neurosci Methods 2015; 254: $1-9$.

20. de Camp N. New methods for extracellular brain recordings in stationary and freely walking honeybees during decision making and virtual navigation. PHILLICA.COM 2013; Article number 373 .

21. Saha D, Leong K, Katta N, Raman B. Multi-unit recording methods to characterize neural activity in the locust (Schistocerca americana) olfactory circuits. J Vis Exp 2013; 71: e50139.

22. Aldworth ZN, Stopfer MA. Trade-off between information format and capacity in the olfactory system. J Neurosci 2015; 35: $1521-1529$.

23. Zhong C, Zhang Y, He W, Wei P, Lu Y, Zhu Y, et al. Multi-unit recording with iridium oxide modified stereotrodes in Drosophila melanogaster. J Neurosci Methods 2014; 222: 218-229.

24. Schultz S. Signal-to-noise ratio in neuroscience. Scholarpedia 2007; 2: 2046.

25. Desai SA, Rolston JD, Guo L, Potter SM. Improving impedance of implantable microwire multi-electrode arrays by ultrasonic electroplating of durable platinum black. Front Neuroeng 2010; 3 : 5.

26. User Manual NanoZ [http://neuralynx.com/products/impedance_ measurement/nanoz].

27. Guo P, Pollack AJ, Varga AG, Martin JP, Ritzmann RE. Extracellular wire tetrode recording in brain of freely walking insects. J Vis Exp 2014; 86: e51337.

28. Stumpner A, Ronacher B. Auditory interneurons in the metathoracic ganglion of the grasshopper Chorthippus biguttulus. J Exp Biol 1991; 158: 391-410.

29. Kutzki O. Kodierung verhaltensrelevanter Gesangsparameter bei Chorthippus biguttulus. PhD thesis. Humboldt Univ zu Berlin. Faculty of Mathematics and Natural science; 2012.

30. Rey HG, Pedreira C, Quiroga RQ. Past, present and future of spike sorting techniques. Brain Res Bull 2015; S0361-9230: 00068-4.

31. Bender JA, Pollack AJ, Ritzmann RE. Neural activity in the central complex of the insect brain is linked to locomotor changes. Curr Biol 2010; 20: 921-926.

32. Mizunami M, Okada R, Yongsheng LI, Strausfeld NJ. Mushroom bodies of the cockroach: Activity and identities of neurons recorded in freely moving animals. J Comp Neurol 1998; 402: 501-519.

33. Guo P, Ritzmann RE. Neural activity in the central complex of the cockroach brain is linked to turning behaviors. J Exp Biol 2013; 216: 992-1002.

34. Ghomashchi A, Zheng Z, Majaj N, Trumpis M, Kiorpes L, Viventi J. A low-cost, open-source, wireless electrophysiology system. In: 36th Annual International Conference of the IEEE Engineering in Medicince and Biology Society 2014: 3138-3141.

35. Harrison RR, Fotowat H, Chan R, Kier RJ, Olberg R, Leonardo A, et al. Wireless neural/EMG telemetry systems for small freely moving animals. IEEE Trans Biomed Circuits Syst 2011; 5: 103-111.

36. Lewicki MS. A review of methods for spike sorting: the detection and classification of neural action potentials. Network 1998; 9: 53-78. 